\title{
Development of a multiple-microhole aerostatic air bearing system
}

\author{
Kuang-Chao Fan ${ }^{1}$, Chi-Chung $\mathrm{Ho}^{1}$ and Jong-I Mou ${ }^{2}$ \\ ${ }^{1}$ Department of Mechanical Engineering, National Taiwan University, Taipei, Taiwan, \\ Republic of China \\ ${ }^{2}$ Department of Industrial Engineering, Arizona State University, Tempe, AZ, USA
}

Received 14 March 2002, in final form 13 May 2002

Published 21 June 2002

Online at stacks.iop.org/JMM/12/636

\begin{abstract}
New types of aerostatic air bearing and linear slide systems have been developed. The prototype of a multiple-microhole, instead of a porous-type, air bearing was developed and the surface roughness was improved by finished process. Instead of the conventional drilling process, the air bearing pads were fabricated by using microfabrication technology in this research. The spectral element method was employed to simulate the pressure distribution of air bearing and a comparison was made with experimental results. A granite straight edge was used as linear slide to guide the moving table that was supported by the developed air bearing system. A laser interferometer system was used to assess the performance of the stage.
\end{abstract}

(Some figures in this article are in colour only in the electronic version)

\section{Introduction}

Air bearing uses the characteristic of viscosity to increase the air pressure at the interface between two objects and to generate the floating effect to sustain payload. As shown in figure 1, based on the pressure generation principles and payload, air bearings can be categorized as: (1) hydrodynamic type-commonly used for low payload applications such as gyros and read-write heads of hard drives; (2) hydrostatic type-commonly used for higher payload applications, such as precision measuring machines and dental drills; (3) squeeze film type-commonly used for damping applications such as in semiconductor accelerometers.

Similar to fluid lubricated bearing, the basic function of air bearing is to provide lubrication and support. Comparing with fluid lubricated bearing, air bearing has lower viscosity and lower sensitivity to temperature variation. Meanwhile, air bearing has the following advantages: (1) no pollution; (2) does not require sealing and cooling; (3) non-sticking. Therefore, the demand for air bearing is increasing for high-speed rotation, precision linear stages, and refrigerator applications.

In the past three decades, the technology of flatbed air bearing has received much attention, and new products have been developed to improve air bearing performance. Several approaches, as shown in figure 2, have been

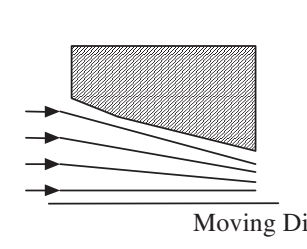

(a)

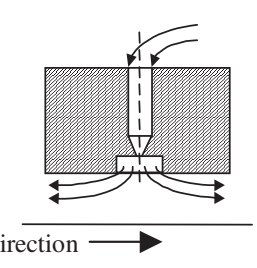

(b)

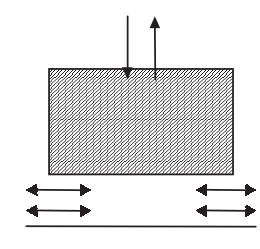

(c)
Figure 1. Various air bearing pressure generation modes. (a) Hydrodynamic, (b) hydrostatic, (c) squeeze film.

adapted to improve the stiffness and stability of flatbed air bearing:

1. adding a compensator or stabilizer such as damping cavity on air bearing $[1,2]$;

2. changing the surface profile of air bearing with grooved bearing [3], arc surface [4], conical shape [5, 6], or more complicated tri-conical gap-shaped holes [7];

3. changing the bearing surface structure or using membrane-type bearing $[5,8]$, or adaptive porous material bearing $[9,10]$.

The purpose of this study is to further improve the characteristics of flatbed aerostatic (hydrostatic) air bearing and to lower its cost. The designed air bearing adopts the 


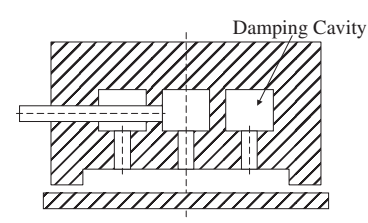

(a)

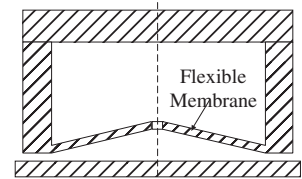

(c)

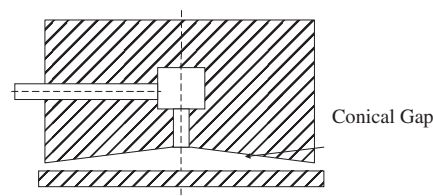

(b)

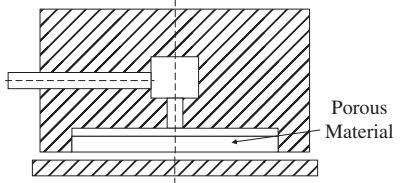

(d)
Figure 2. Various air bearing design schemes.

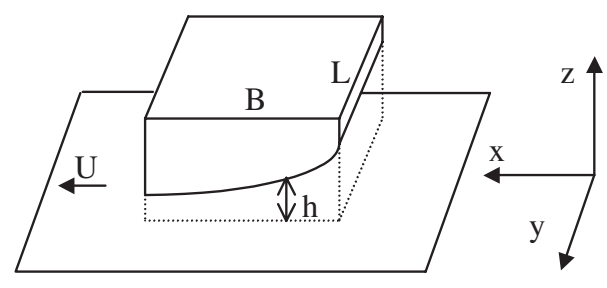

Figure 3. Air bearing sliding mode.

multiple-hole concept. The goals are to bring the pressure to extend location without using a groove structure and to maintain a stable airflow effect. The multiple-hole structure allows a better stability in airflow. Meanwhile, compared with porous material air bearing, the fly height can be reduced due to finer surface roughness. To achieve airflow restriction, the size of the hole cannot be too large. Therefore, we need to consider the hole size and the number of holes when designing the air bearing pad and the corresponding fabrication process.

The conventional method of producing microholes of the air pad has been to apply a drilling process on the metal plate. The thin drill is subject to wear and break causing poor quality of the holes. In addition, the hole diameter is limited by the drill size. A new technology employing an advanced microelectromechanical systems (MEMS) process has, therefore, been used to produce multiple-microhole air bearing. The spectral element method has been used to analyze the designed air bearing pressure distribution. The analytical result has been compared with experimental data and validated on a linear sliding system.

\section{Analytical analysis and modeling}

Theoretically, the airflow characteristic at the air bearing and sliding guideway interface dominates the air bearing performance. Although the fly height of most air bearings is less than $20 \mu \mathrm{m}$, the continuous airflow condition is still validated [11].

Figure 3 depicts a typical sliding mode of an air bearing, where $B$ is the length in the $x$-direction, $L$ is the width in the $y$-direction, $U$ is the velocity along the $x$-direction, $h$ is the fly height, $P$ is the local pressure, and $\rho$ is the density. We assume that the ambient temperature $T$ is constant, $B \approx L \approx$ $1, h \approx 10^{-4}$, the velocity in the $z$-direction is neglected, the viscosity $(\mu)$ dominated by $\frac{\partial^{2} u}{\partial z^{2}}$ and $\frac{\partial^{2} v}{\partial z^{2}}$, and laminar flow at the interface. Thus

$$
\frac{\partial P}{\partial x}=\frac{\partial}{\partial z}\left(\mu \frac{\partial u}{\partial z}\right), \quad \frac{\partial P}{\partial y}=\frac{\partial}{\partial z}\left(\mu \frac{\partial v}{\partial z}\right) .
$$

According to the boundary conditions, $u=U$ and $v=0$ at $z=0, u=0$ and $v=0$ at $z=h$, this yields

$$
\begin{gathered}
u=\frac{1}{2 \mu}\left(\frac{\partial P}{\partial x}\right)\left(z^{2}-h z\right)+\frac{U}{h}(h-z), \\
v=\frac{1}{2 \mu}\left(\frac{\partial P}{\partial y}\right)\left(z^{2}-h z\right) .
\end{gathered}
$$

Since $\frac{\partial \rho}{\partial t}+\frac{\partial}{\partial x}(\rho u)+\frac{\partial}{\partial y}(\rho v)+\frac{\partial}{\partial z}(\rho w)=0$ and $\frac{P}{\rho}=g R T$, by taking the integral of $\int_{0}^{h}\left[\frac{\partial}{\partial x}(\rho u)+\frac{\partial}{\partial y}(\rho v)\right] \mathrm{d} z+\frac{\partial}{\partial t}(\rho h)=0$ and substituting $u$ and $v$, the Reynolds equation can be derived as follows:

$$
\frac{\partial}{\partial x}\left(\frac{\rho h^{3}}{\mu} \frac{\partial P}{\partial x}\right)+\frac{\partial}{\partial y}\left(\frac{\rho h^{3}}{\mu} \frac{\partial P}{\partial y}\right)=6\left\{U \frac{\partial}{\partial x} \rho h+2 \frac{\partial}{\partial t} \rho h\right\} .
$$

For aerostatics air bearing airflow analysis, $\frac{\partial}{\partial t}(\rho h)=0$ when a stable airflow condition exists and $U \frac{\partial}{\partial x}(\rho h)=0$ when the relative velocity of the air bearing is low. Therefore, the terms on the right-hand side of equation (1) can be neglected and this equation can be simplified as

$$
\frac{\partial}{\partial x}\left(\frac{\rho h^{3}}{\mu} \frac{\partial P}{\partial x}\right)+\frac{\partial}{\partial y}\left(\frac{\rho h^{3}}{\mu} \frac{\partial P}{\partial y}\right)=0 .
$$

Its boundary conditions are the given restrictor outlet pressure and ambient pressure.

Next, the developed spectral element method [12], which combines the finite element and spectral methods, was used to calculate the pressure distribution index. First, the IDEAS CAD system was used to generate meshes for the air bearing pad surface. The mesh information was modified into a data file format that is readable by a program based on the spectral element method. Tecplot graphic software was then used to plot the calculated air bearing pressure distribution profile. The simulation flow chart is shown in figure 4.

Using the developed approach, the pressure distributions of several air bearing designs were simulated. For each mesh, we needed to provide the coordinates of four corners and the boundary conditions of four edges. The ambient pressure and restrictor outlet pressure were specified but the pressure between internal meshes was relative.

Figure 5 shows the simulated air pressure distribution for an air bearing surface comprising 25 square microholes, each with a cross-sectional area of $30 \mu \mathrm{m}^{2}$. The given boundary conditions such as the ambient pressure and the restrictor outlet pressure are set to 1 and 3 atmospheres, respectively.

\section{MEMS technology for air bearing fabrication}

Silicon is the most commonly used material in modern microfabrication processes. The major reason is because silicon has excellent and stable mechanical characteristics. Its Young's modulus $(E=130-190 \mathrm{GPa})$ and hardness $(H=850)$ 


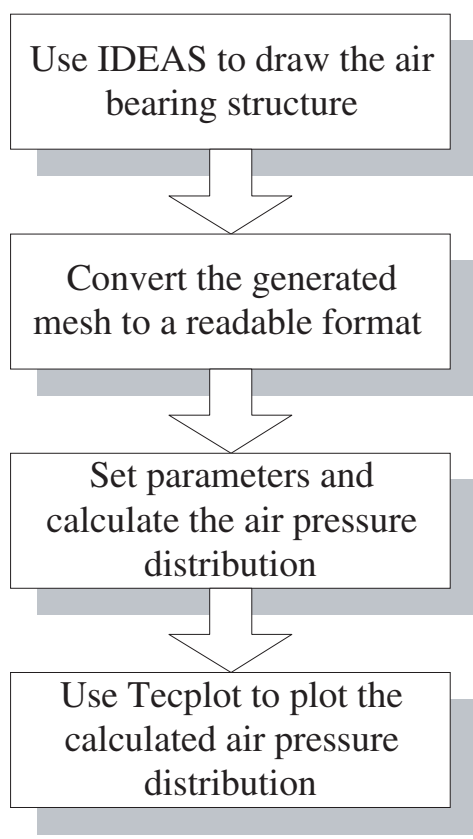

Figure 4. Air pressure simulation flow chart.

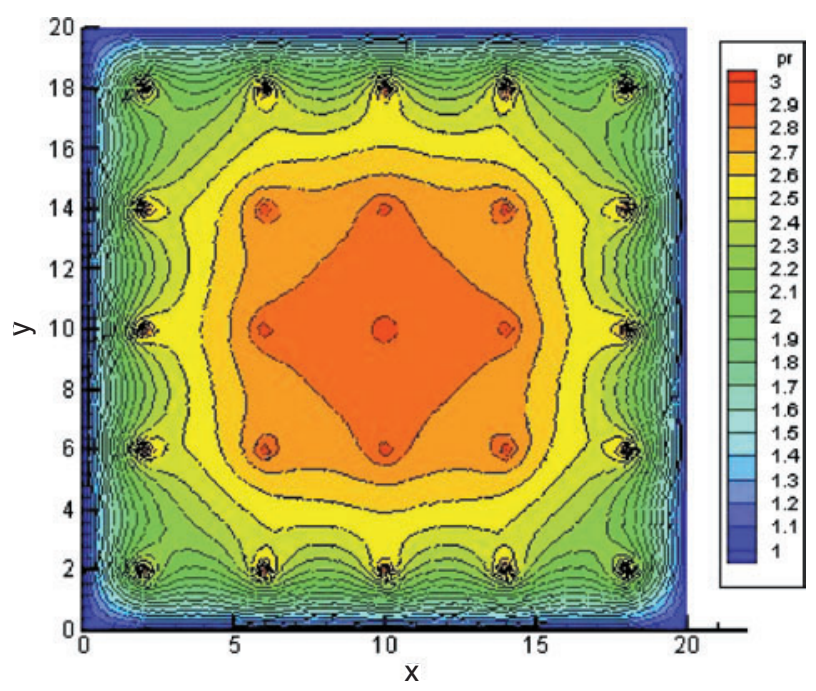

Figure 5. Simulated air pressure distribution for a surface comprising 25-30 $\mu \mathrm{m}^{2}$ microholes.

are similar to those of steel $(E=200 \mathrm{GPa}, H=660)$ and it has a small Poisson's ratio, $v \approx 0.18$. On the other hand, silicon is very easy to break along the natural fracture plane and it is difficult to fabricate it into a three-dimensional profile.

\subsection{Mask design}

In this study, we have used a (100) double-side polished four inch silicon wafer. The use of anisotropic etching produces a (111) etching plane, as shown in figure 6. Therefore, we need to consider the angle formed during etching and the silicon wafer thickness when designing the mask hole diameter. The

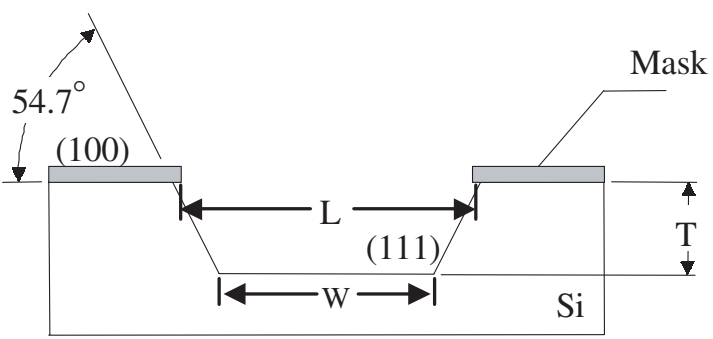

Figure 6. Anisotropic etching of (100) silicon.
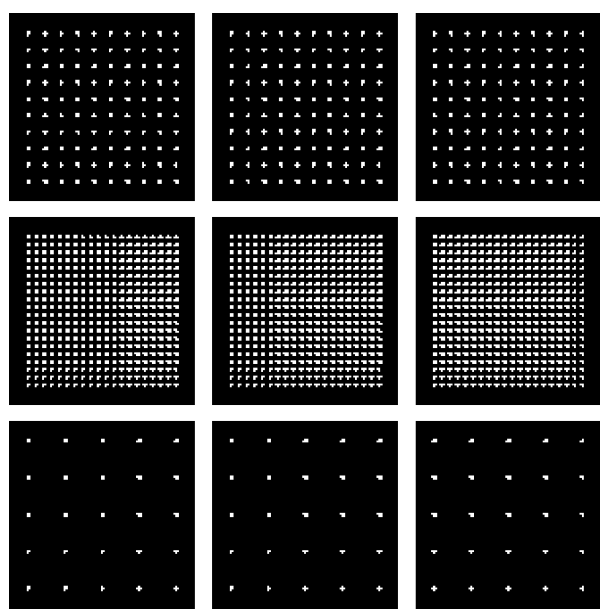

Figure 7. Configuration of a mask design.

following relationship was thus adopted

$$
L=W+2 T \cot 54.7^{\circ}
$$

where $W$ is the bottom edge length of the etched structure, $L$ is the edge length of the mask and $T$ is the thickness of the etched structure.

If the restrictor hole size $(W)$ is $10 \mu \mathrm{m}$ and the thickness $(T)$ of the (100) silicon wafer is $500 \mu \mathrm{m}$, since the (111) plane and the bearing surface have a $54.7^{\circ}$ angle relationship, the length $(L)$ can be calculated as $718 \mu \mathrm{m}$. Therefore, as shown in figure 7, the mask was designed to have 25,100 and 400 holes with $L$ equal to 713,718 and $723 \mu \mathrm{m}$, respectively.

\subsection{MEMS processes}

A round-shaped hole was adopted in designing the mask so that the difficulty of aligning a square hole edge to the etching line could be avoided. Due to the characteristics of anisotropic etching, the etching rate along the (100) plane is much faster than the etching rate along the (111) plane. Therefore, although the etching starts with round-shaped hole, the etched hole gradually become a square hole and the etching process progresses along the angular surface of the (111) plane and etch through the silicon wafer.

During the $\mathrm{KOH}$ wet etching process, the observed etching ratio between the (100) and (111) planes is about $1: 20$. To achieve a $30 \mu \mathrm{m}$ microhole outlet, the mask design was then modified to have $L$ equal to $670 \mu \mathrm{m}$ for the pattern of 25 holes. The silicon wafer thickness was selected as $500 \mu \mathrm{m}$ 


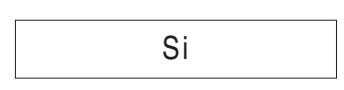

(a)

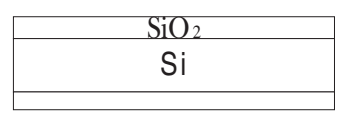

(b)

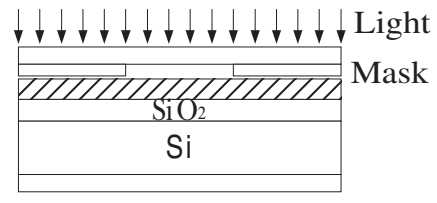

(c)

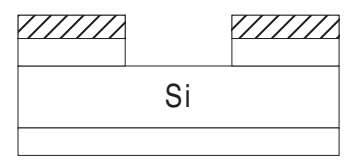

(d)

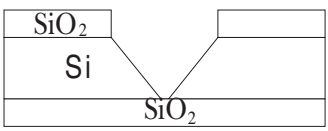

(e)

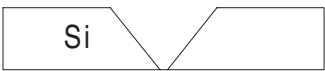

(f)

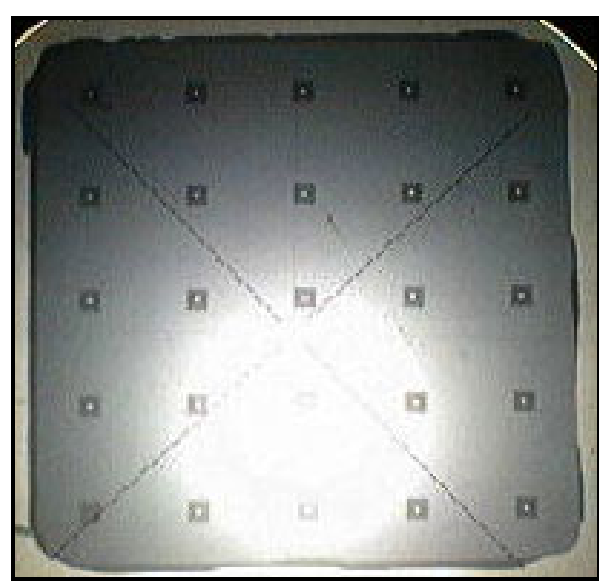

Figure 9. Finished microhole product.

Figure 8. Microfabrication flow chart.

to withstand higher air pressure. Lapping and polishing were used to obtain the desired thickness. The microfabrication process is outlined in figure 8 .

The silicon wafer was pre-baked for $30 \mathrm{~min}$ to remove the surface moisture and to increase the adhesion of the photoresist. The photoresist was spin-coated on to the wafer surface with $4000 \mathrm{rpm}$ for $40 \mathrm{~s}$ to achieve a $1.2 \mu \mathrm{m}$ coating thickness. The FH-6400L positive photoresist was used for its good resolution. The coated wafer was soft baked in a $120{ }^{\circ} \mathrm{C}$ oven for $10 \mathrm{~min}$. Photolithography and the designed mask were used to transfer the mask pattern on to the photoresist. A solution of FHD-5 was used to develop the exposed photoresist. The wafer was then hard baked in a $120{ }^{\circ} \mathrm{C}$ oven for $20 \mathrm{~min}$.

In this study, silicon and silicon oxide are the two major materials that need to be etched out to form the microhole. A buffered oxide etch (BOE) solution was used to etch silicon dioxide. The etching rate was about $120 \mathrm{~nm} \mathrm{~min}{ }^{-1}$. To meet the requirements for etching a large volume of silicon material and for a high accuracy of the etched structure, $\mathrm{KOH}$ was used for anisotropic etching.

BOE was first used to etch the exposed silicon dioxide $\left(\mathrm{SiO}_{2}\right)$ that was not covered by the photoresist material. $\mathrm{KOH}$ was then used to etch out the unwanted silicon material that was not covered by the $\mathrm{SiO}_{2}$. Since etchant would attack silicon wafer indiscriminately, thus silicon dioxide was used as the protection layer to cover the silicon substrate surface that was not etched. The etched hole geometry depends on the pattern of etched silicon dioxide. Both the etching temperature and $\mathrm{KOH}$ solution concentration will affect the etching rate of silicon and silicon dioxide. The relative etching ratio between silicon and silicon dioxide should be carefully controlled during the etching process.

As the thicknesses of the silicon dioxide and silicon substrates were 1.5 and $500 \mu \mathrm{m}$ respectively, the thickness ratio was about $1: 333$. A $\mathrm{KOH}$ solution of a $20 \%$ concentration by weight and a temperature of $80{ }^{\circ} \mathrm{C}$ were selected to anisotropically etch the silicon. The etching rate was about $85 \mu \mathrm{m} \mathrm{h}^{-1}$. The etching temperature was then decreased to $60^{\circ} \mathrm{C}$ to improve the yield and product quality but

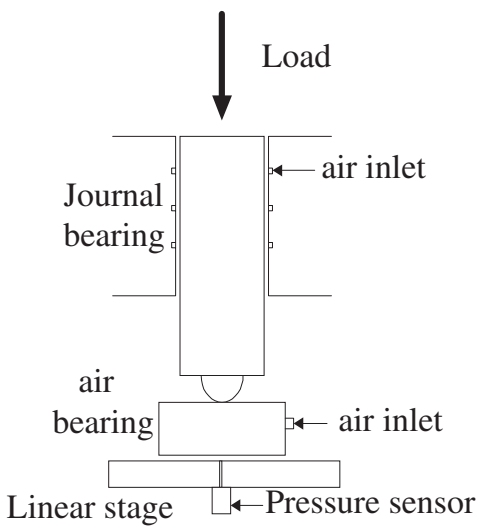

Figure 10. Pressure testing system configuration.

with the trade-off of longer etching time. An etched microhole product is shown in figure 9.

\section{Air pressure measuring experiment}

According to Holster et al [4], Holster and Jacobs [8], Boffey and Desai [13] and Fukui and Kaneko [14], an air bearing pressure distribution measuring system was built to test the air bearing characteristics such as pressure distribution and stiffness. The testing structure needs to be able to sustain a certain load. It also needs to have a moving platform with position control capability and a pressure sensing device, as shown in figure 10.

The testing structure is basically a ring structure. A platform consisting of a two-axis linear stage was placed at the center to provide the movement during the pressure measurement. The platform must meet the demands of high load capacity and position accuracy. The platform was made of steel material with both sides ground and then chrome-plated to achieve the effect of polishing and corrosion resist.

A $0.5 \mathrm{~mm}$ diameter hole was generated using electrical charge machining at the center of the platform. A pressure tube was first inserted into the hole and then connected to a pressure sensor to measure the pressure distribution at the interface 


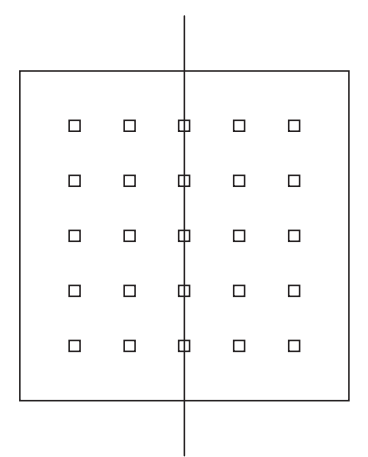

Cross Restrictors

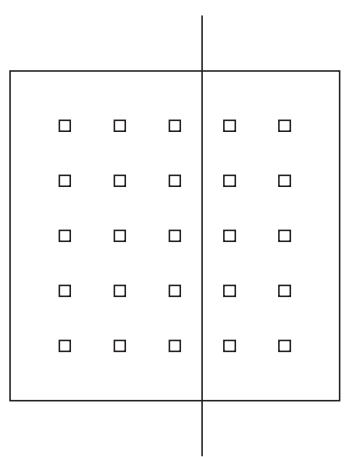

Between Restrictors

Figure 11. Pressure measurement scheme.

Pressure Distribution at the Line Cross Restrictors

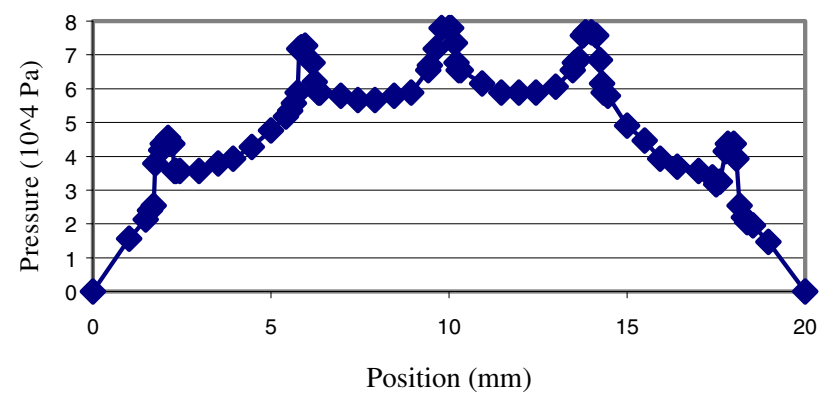

Figure 12. Cross-restrictor pressure distribution of type 1 air bearing.

between the air bearing and the platform. The pressure sensor operation range is $0.3923 \mathrm{MPa}$ with a $0.0196 \mathrm{MPa}$ resolution.

The pressure distribution was measured for both crossrestrictor and between-restrictor situations as shown in figure 11. The measurement was conducted for two types of air bearing design. The first type of air bearing has 25 holes with a $80 \mu \mathrm{m}^{2}$ opening. The second type of air bearing has 400 holes with a $30 \mu \mathrm{m}^{2}$ opening. The cross-restrictor and between-restrictor pressure distributions of the type 1 air bearing are shown in figures 12 and 13, respectively. The cross-restrictor and between-restrictor pressure distributions of the type 2 air bearing are shown in figures 14 and 15 , respectively.

The results show that, for the type 1 air bearing, the pressure at each restrictor is different and there is a significant pressure drop in the proximity of the restrictor. However, there is no such pressure drop in the proximity of the restrictor for the type 2 air bearing. The pressure distribution at regions between restrictors is relatively smooth for both types of air bearing.

Therefore, as the hole size decreases and the number of holes increases, the pressure drop at the restrictor decreases and could eventually reach a smooth and uniform pressure distribution. The higher the pressure level in each unit area, the higher the load the bearing can sustain.

The experimental results were compared with the simulation results derived from using the spectral element

Pressure Distribution at the Line between Restrictors

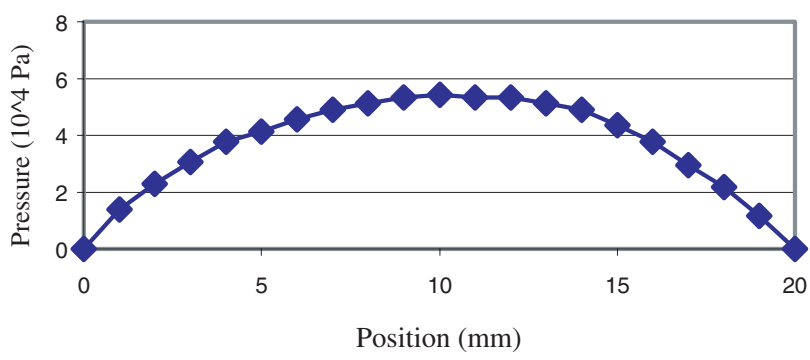

Figure 13. Between-restrictor pressure distribution of type 1 air bearing.

Pressure Distribution at the Line Cross Restrictors

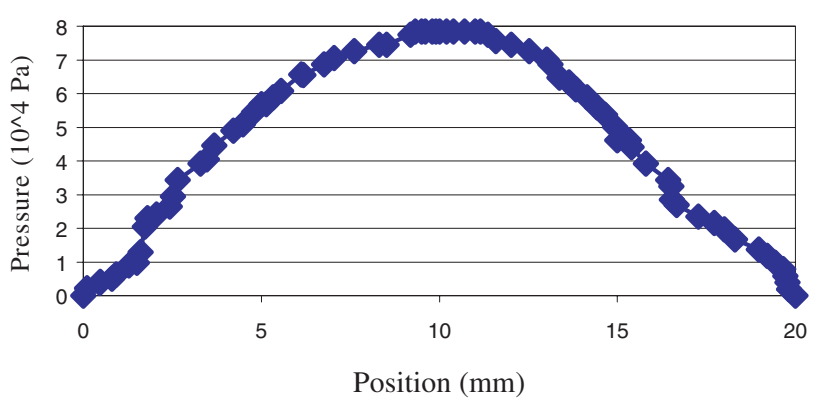

Figure 14. Cross-restrictor pressure distribution of type 2 air bearing.

Pressure Distribution at the Line between Restrictors

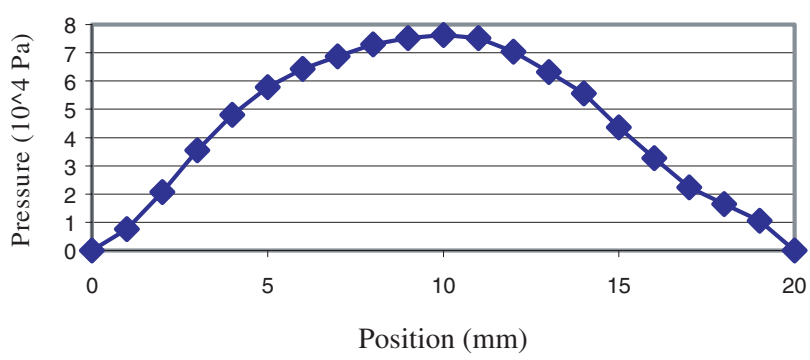

Figure 15. Between-restrictor pressure distribution of type 2 air bearing.

method. The boundary conditions used for the simulation are set as follows. The restrictor outlet pressure is $0.0599 \mathrm{MPa}$, the ambient pressure is 1 atmosphere, the input pressure is $0.0981 \mathrm{MPa}$, and the load is $1140 \mathrm{~g}$. The calculated output pressure for the cross restrictors is about $0.0736 \mathrm{MPa}$. Compared with the experimental results for both types of air bearing depicted in figures 12 and 14, the actual pressure distribution for cross restrictors do not have the same uniform pressure distribution pattern derived from simulation, as shown in figures 16 and 17. Although the difference between the simulated and experimental results changes slightly, the 


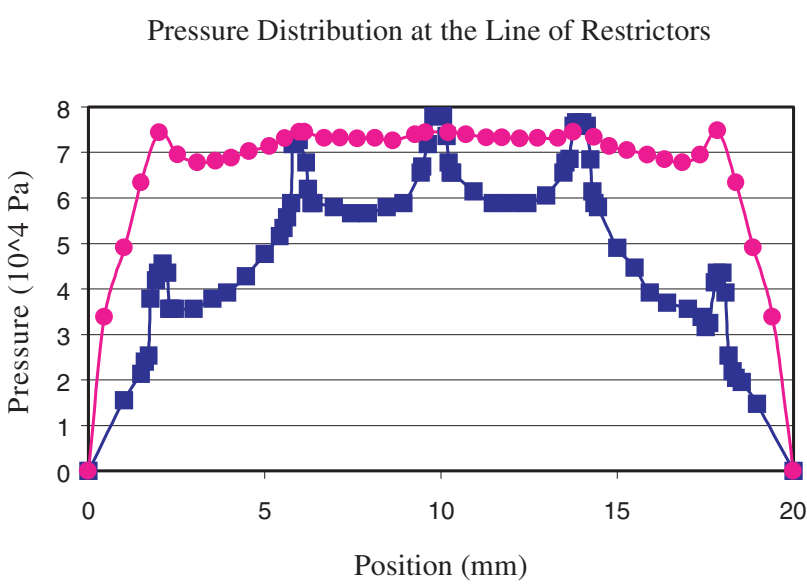

Figure 16. Simulated and experimental pressure distribution for the type 1 air bearing.

Pressure Distribution at the Line of Restrictors

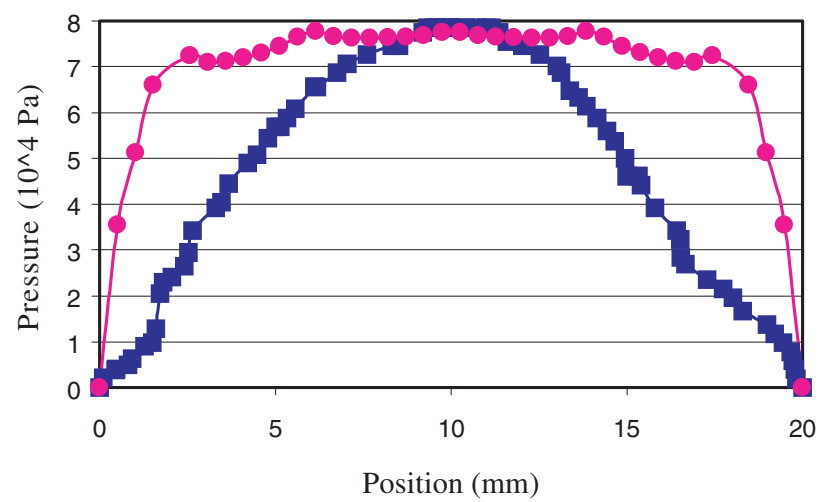

Figure 17. Simulated and experimental pressure distribution for the type 2 air bearing.

pressure distribution for the type 2 air bearing becomes smoother with an increased number of holes as the pressure is evenly distributed.

Stiffness is another major characteristic in air bearing performance analysis. A linear variable differential transformer (LVDT) was installed on the same platform for pressure measurement to analyze the relationship between payload and fly height. The results show that there is an inverse relationship between the payload and fly height, as shown in figures 18 and 19 . The slope of the fly height versus payload curve represents the stiffness of the air bearing. The experimental results show that both types of air bearing have similar stiffness. Since the payload depends on the actual supporting area of the air bearing pad, therefore, with same input air pressure, the type 2 air bearing can sustain a higher payload at the same fly height due to a larger number of restrictors area than the type 1 air bearing. Meanwhile, the more holes there are, the better the stability of the air bearing pad. Comparing the pressure distributions between figures 12 and 14 as well as between figures 13 and 15 and then integrating the areas under the distributions, we can also derive the same conclusion. The larger the integrated area

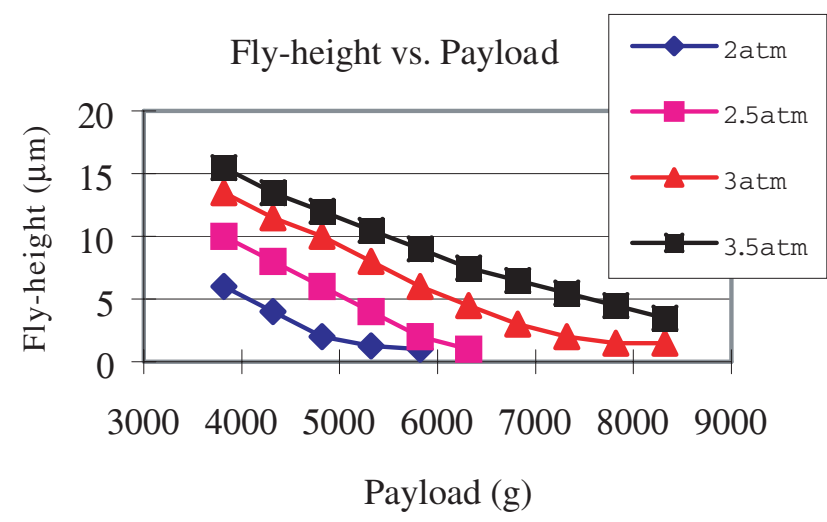

Figure 18. Fly height versus payload of the type 1 air bearing.

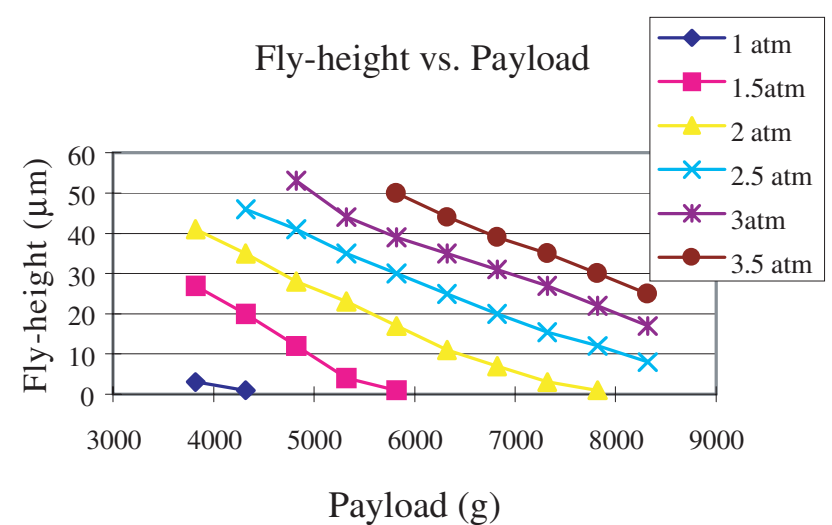

Figure 19. Fly height versus payload of the type 2 air bearing.

under the pressure distribution curve, the higher the sustainable payload.

\section{Air floating guideway stability analysis}

In general, an air floating guideway can provide a higher precision motion than a contacting guideway. With the assistance of compressive airflow, the moving platform can travel on the guideway without contact. The effect of guideway surface roughness and imperfection can be slightly smoothed out. Meanwhile, the friction force is almost equal to zero. Thus, the platform can move more responsively.

Depending on the relative structure between the guideway and the platform, the platform equipped with aerostatic air bearing can be categorized as either an open or a closed structure. Figure 20 shows four different types of air floating guideway that are commonly used for precision equipment such as coordinate measuring machines (CMMs) and positioning stages.

Using the previously designed air bearing and adopting the close weight balance type structure, an air floating guideway was developed as shown in figure 21. A U-shaped guide and six air bearings were assembled to form the sliding platform.

A laser interferometer system was used to measure the linear motion straightness of the developed sliding system. 


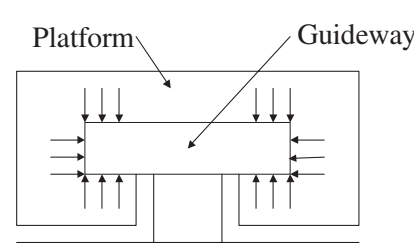

(a)

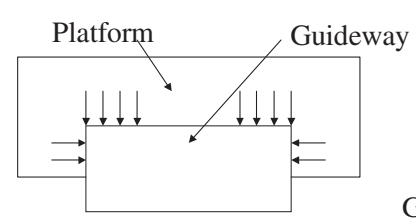

(c)

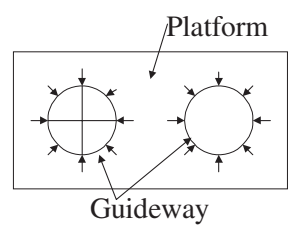

(b)

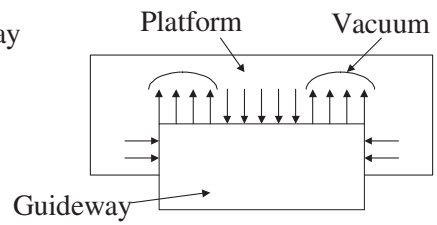

(d)

Figure 20. Various air floating guideway structures.

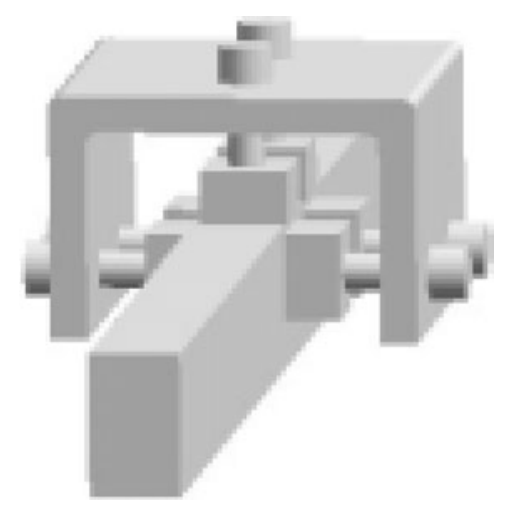

Figure 21. Air floating bearing assembly scheme.

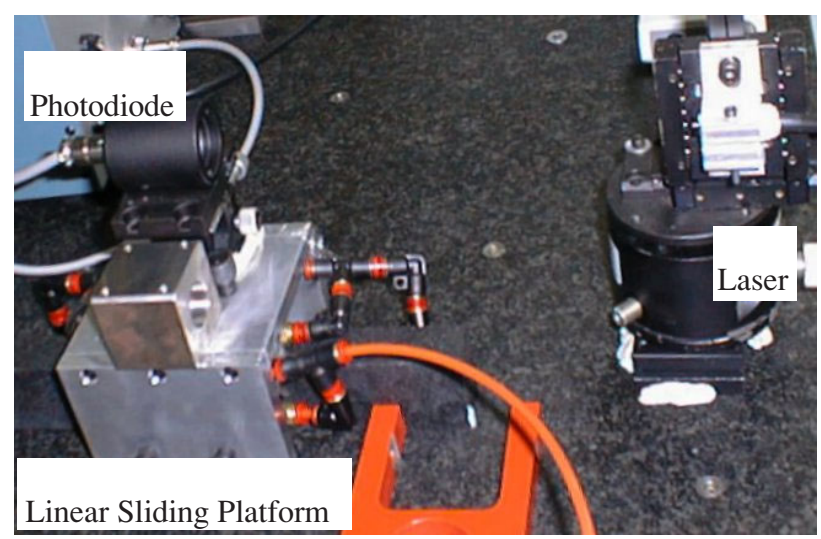

Figure 22. Straightness error measurement setup.

The experimental setup is shown in figure 22. The measured straightness in the $x$ - and $y$-directions is shown in figure 23 and 24 , respectively.

The potential reasons for larger straightness error in the $x$-direction could be summarized as follows.

1. It is difficult to fine-tune the air bearing to the location for an optimal air gap between the air bearing and the granite sliding structure. Difficulty may occur during sliding if the air gap is too small. On the other hand, a larger straightness error in the $x$-direction may occur if the air gap is too large, as shown in figure 25 .
Straightness

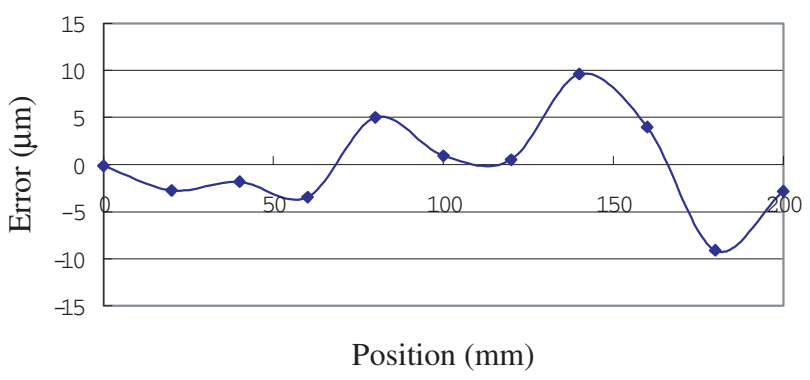

Figure 23. Linear slide straightness error in the $x$-direction.

Straightness

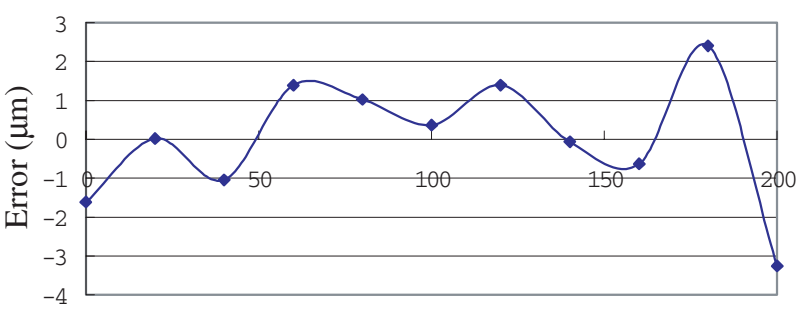

Position (mm)

Figure 24. Linear slide straightness error in the $y$-direction.

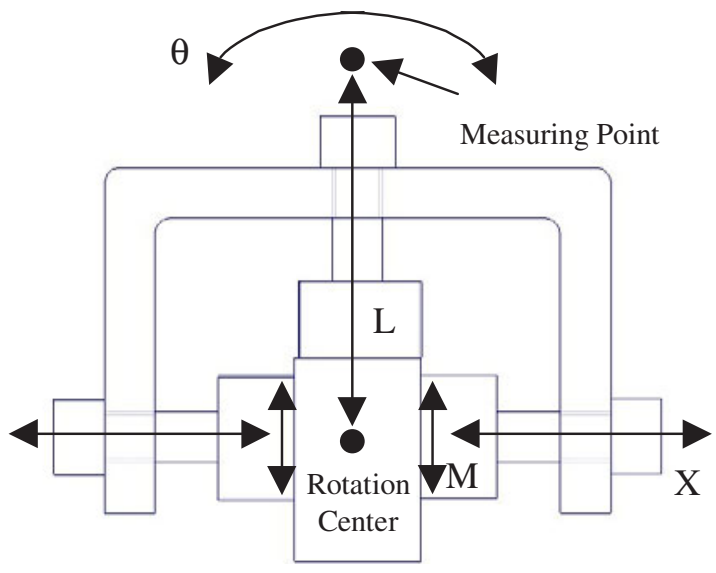

Figure 25. Straightness error effect in the $x$-direction.

2. A slight rotation $(\theta)$ may occur if the platform is subject to a torque $(M)$ as shown in figure 25 . The product of $L$ and $\theta$ will induce further straightness error in the $x$-direction.

\section{Conclusions}

In this research, a multiple-microhole air bearing and linear slide were developed. The air bearing pads were fabricated by using microfabrication technology. The multiple-hole structure allows a better stability in airflow. Meanwhile, compared with porous material air bearing, the fly height can be reduced due to finer surface roughness. The spectral element method was employed to analyze and simulate the pressure distribution of the designed air bearing. 
The analytical result was compared with experimental data and validated on a linear sliding system. A granite straight edge was used as linear slide to guide the moving table supported by the developed air bearing system. A laser interferometer system was used to assess the performance of the stage.

Modern MEMS technology was applied to fabricate micro diameter holes. Although parameter identification and process control are very time consuming, the prototype air bearing products exhibit good mechanical characteristics and performance.

\section{References}

[1] Haruo M and Atsunobu M 1967 On the stabilizing methods of externally pressurized thrust gas bearings J. Lubr. Technol. 89 283-90

[2] Stowell T B 1971 Pneumatic hammer in a gas lubricated externally pressurized annular thrust bearing J. Lubr. Technol. 93 498-503

[3] Fuller D D 1959 General review of gas-bearing technology Proc. 1st Int. Symp. on Gas Lubricated Bearings pp 1-27

[4] Holster P, Jacobs J and Roblee J 1991 The measurement and finite element analysis of the dynamic stiffness of non-uniform clearance, gas, thrust bearings Trans. ASME $113768-76$
[5] Bloudeel E, Snoeys R and Devieze L 1976 Aero-static bearing with variable gap configuration Proc. 7th Int. Symp. on Gas Bearing paper-E2

[6] Plessers P and Snoeys R 1988 Dynamic identification of convergent externally pressurized gas-bearing gaps J. Tribol. $110263-70$

[7] Wang J M 1993 Design of gas bearing system for precision application PhD Dissertation Technical University Eindhoven, The Netherlands

[8] Holster P L and Jacobs J A H 1987 Theoretical analysis and experimental verification on the static properties of externally pressurized air bearing pads with load compensation Tribology $20276-89$

[9] Sneck H J 1968 A survey of gas-lubricated porous bearings J. Lubr. Technol. 90 804-9

[10] Sun D C 1973 Stability analysis of an externally pressurized gas lubricated porous thrust bearing J. Lubr. Technol. 95 457-68

[11] Gross W A 1962 Gas Film Lubrication (New York: Wiley)

[12] Chen X W 1996 Application of spectral element method in flame combustion MS Thesis Department of Mechanical Engineering, National Taiwan University

[13] Boffey D A and Desai D M 1980 An experimental investigation into rubber stabilization of an externally pressurized air-lubricated thrust bearing J. Lubr. Technol. $10265-70$

[14] Fukui S and Kaneko R 1988 Experimental investigation of externally pressurized bearings under high knudsen number conditions Trans. ASME 110 144-7 